This item was submitted to Loughborough's Research Repository by the author.

Items in Figshare are protected by copyright, with all rights reserved, unless otherwise indicated.

\title{
Combination quantum oscillations in canonical single-band Fermi liquids
}

PLEASE CITE THE PUBLISHED VERSION

PUBLISHER

(C) American Physical Society

VERSION

VoR (Version of Record)

LICENCE

CC BY-NC-ND 4.0

REPOSITORY RECORD

Alexandrov, A.S., and V.V. Kabanov. 2019. "Combination Quantum Oscillations in Canonical Single-band Fermi Liquids”. figshare. https://hdl.handle.net/2134/4247. 
This item was submitted to Loughborough's Institutional Repository (https://dspace.lboro.ac.uk/) by the author and is made available under the following Creative Commons Licence conditions.

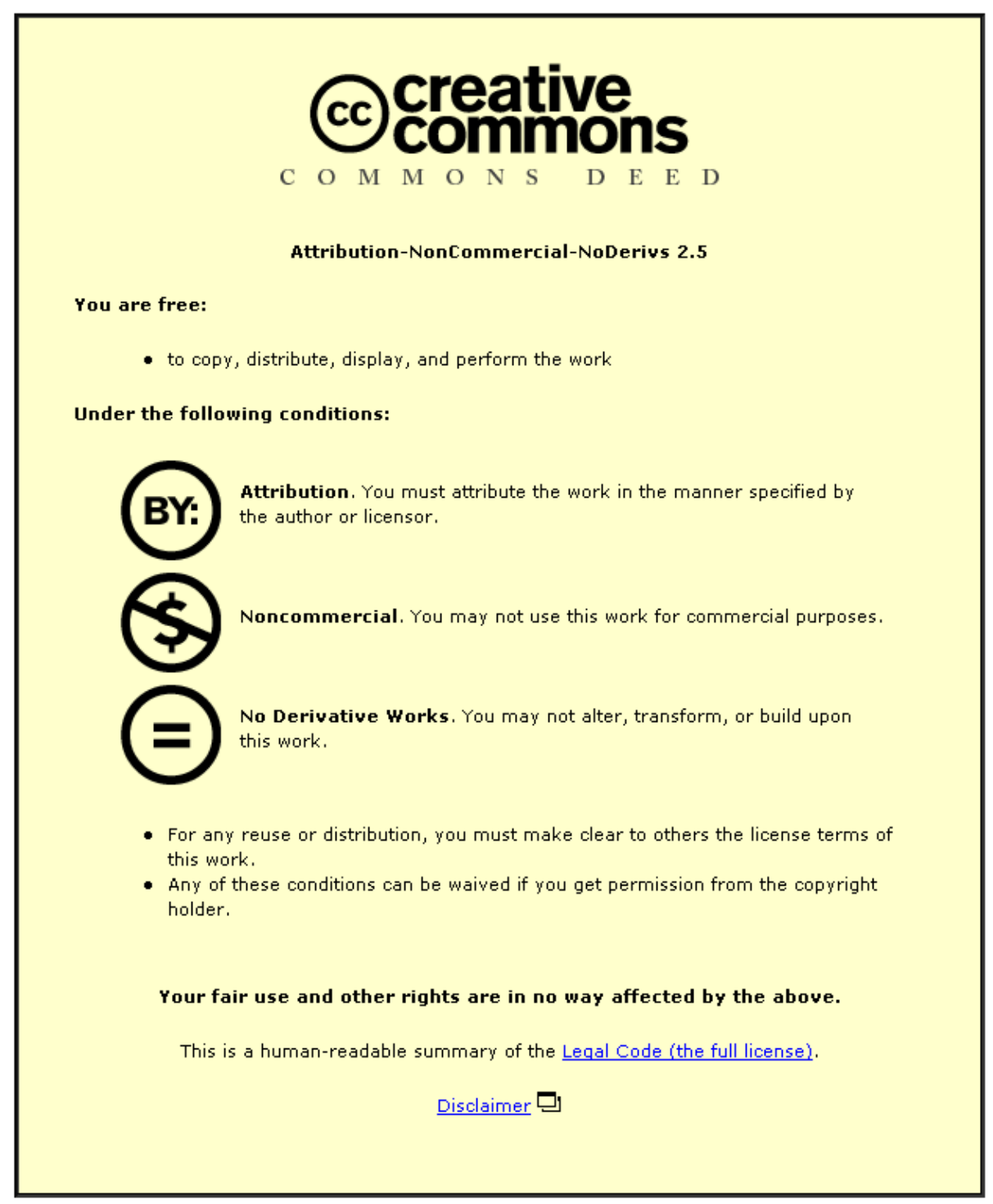

For the full text of this licence, please go to: http://creativecommons.org/licenses/by-nc-nd/2.5/ 


\title{
Combination quantum oscillations in canonical single-band Fermi liquids
}

\author{
A. S. Alexandrov ${ }^{1}$ and V. V. Kabanov ${ }^{2}$ \\ ${ }^{1}$ Department of Physics, Loughborough University, Loughborough LE11 3TU, United Kingdom \\ ${ }^{2}$ Josef Stefan Institute 1001, Ljubljana, Slovenia
}

(Received 3 August 2007; published 5 December 2007)

\begin{abstract}
Chemical potential oscillations mix individual-band frequencies of the de Haas-van Alphen and Shubnikov-de Haas magnetooscillations in canonical low-dimensional multiband Fermi liquids. We predict a similar mixing in canonical single-band Fermi liquids, which Fermi surfaces have two or more extremal cross sections. Combination harmonics are analyzed using a single-band almost two-dimensional energy spectrum. We outline some experimental conditions allowing for resolution of combination harmonics.
\end{abstract}

DOI: 10.1103/PhysRevB.76.233101

PACS number(s): 72.15.Gd, 75.75.+a, 73.63.Nm

Magnetic quantum oscillations of magnetization [de Haas-van Alphen (dHvA) effect] and resistivity [Shubhikov-de Haas (SdH) effect] are unequivocal hallmarks of the Fermi liquid, providing most reliable and detailed Fermi surfaces, ${ }^{1}$ in particular, in layered organic metals $^{2,3}$ and almost two-dimensional (2D) superconductors such as $\mathrm{Sr}_{2} \mathrm{RuO}_{4}{ }^{4}$ An interesting feature of $\mathrm{dHvA}$ and/or $\mathrm{SdH}$ oscillations is a difference between canonical and grand canonical ensembles. While it is negligible in threedimensional metals, ${ }^{5}$ the quantum fluctuations of two thermodynamically identical ensembles are very different in 2D multiband metals. In particular, there are combination frequencies in dHvA and/or $\mathrm{SdH}$ oscillations of a 2D multiband metal with fixed electron density $n_{e}$ (canonical ensemble), predicted by Alexandrov and Bratkovsky (AB), ${ }^{6}$ and studied numerically ${ }^{6-11}$ and analytically. ${ }^{12-14}$ The effect has been experimentally observed in different low-dimensional systems. ${ }^{3,15,16}$ Obviously, there are no mixing of the fundamental single-band frequencies, if the chemical potential $\mu$ is fixed by a reservoir (grand-canonical ensemble). Importantly, samples are normally placed on nonconducting substrates with no electrodes attached, so the system is closed in actual dHvA experiments.

As it happens the fundamental frequency mixing due to the chemical potential oscillations (AB effect) may be obscured by mixing due to the magnetic breakdown ${ }^{17}$ (MB effect), as discussed by Kartsovnik. ${ }^{3}$ The MB effect is the switching of two close electron orbits in different bands on the Fermi surface (FS) at sufficiently strong magnetic fields. Here, we predict a mixing of two or more fundamental frequencies in a canonical single-band Fermi liquid with a few extremal FS cross sections, where the MB is nonexistent.

To illustrate the point, we consider an anisotropic single band, with the dispersion $E(\mathbf{k})$ in zero magnetic field,

$$
E(\mathbf{k})=\frac{\hbar^{2} k_{\|}^{2}}{2 m}-2 t_{\perp} \cos \left(k_{\perp} d\right),
$$

which is a fair approximation for a band in layered metals.,3 Here, $k_{\|}$and $k_{\perp}$ are the in-plane and out-of-plane quasimomenta, $t_{\perp}$ is the interplane hopping integral, and $d$ is the interplane distance.

When the magnetic field $B$ is applied, the spectrum [Eq. (1)] is quantized as ${ }^{18}$

$$
E_{n}\left(k_{\perp}\right)=\hbar \omega_{c}(n+1 / 2)-2 t \cos \left(k_{\perp} d\right) \pm g \mu_{B} B / 2,
$$

where $\omega_{c}=e B \cos (\Theta) / m$ is the cyclotron frequency (n $=0,1,2, \ldots), t=t_{\perp} J_{0}\left[k_{F} d \tan (\Theta)\right]\left[J_{0}(x)\right.$ is the Bessel function], $\Theta$ is the angle between the field and the normal to the planes, $g$ is the electron $g$ factor, and $\mu_{B}$ is the Bohr magneton. The spectrum [Eq. (2)] is perfectly $2 \mathrm{D}$ at the Yamaji angles $^{19}$ found from $J_{0}\left[k_{F} d \tan (\Theta)\right]=0$, where $\hbar k_{F}$ $=(2 m \mu)^{1 / 2}$ is the Fermi momentum in pure $2 \mathrm{D}$ case, but otherwise there are two extremal semiclassical orbits. They give rise to beats in $\mathrm{dHvA} / \mathrm{SdH}$ oscillations with two fundamental Fourier-transform (FT) frequencies, $F_{l, s}$ $=\hbar A_{l, s} / 2 \pi e \cos (\Theta)$, revealing modulations of the cylindrical FS along the perpendicular direction (Fig. 1), as observed, e.g., in $\mathrm{Sr}_{2} \mathrm{RuO}_{4}{ }^{4}, 16$

Since there are no different bands, one might expect neither $\mathrm{AB}$ nor $\mathrm{MB}$ mixing of the fundamental frequencies $F_{l}$ and $F_{s}$ in the single-band model [Eq. (1)], in contrast with canonical multiband systems. ${ }^{6,17}$ Actually, as we show below, $F_{l}$ and $F_{s}$ turn out mixed, if $n_{e}$ is constant, so that a combination frequency $F_{+}=F_{l}+F_{s}$ appears similar to the $\mathrm{AB}$ combination frequency ${ }^{6}$ in two-band canonical Fermi liquids. Using conventional Poisson's summation and integrals, ${ }^{1}$ the grand canonical potential per unit volume,

$$
\Omega=-\frac{k_{B} T e B \cos (\Theta)}{4 \pi^{2} \hbar} \sum_{n} \int_{-\pi / d}^{\pi / d} d k_{\perp} \ln \left[1+e^{\left[\mu-E_{n}\left(k_{\perp}\right)\right] / k_{B} T}\right],
$$

is given by $\Omega=\widetilde{\Omega}-m \mu^{2} / 2 \pi d \hbar^{2}$, where

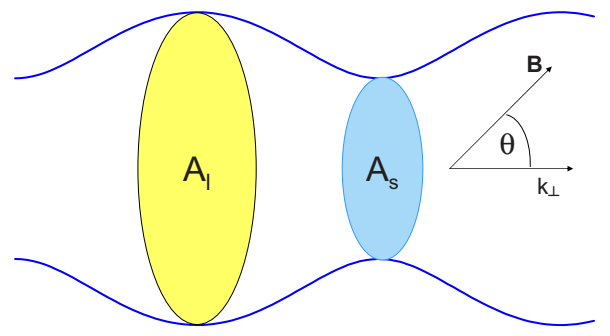

FIG. 1. (Color online) Large, $A_{l}=2 \pi m(\mu+2 t) / \hbar^{2}$, and small, $A_{s}=2 \pi m(\mu-2 t) / \hbar^{2}$, extremal cross sections of a layered-metal Fermi surface. 


$$
\begin{aligned}
\widetilde{\Omega}= & \frac{e^{2} B^{2} \cos ^{2}(\Theta)}{4 \pi^{3} m d} \sum_{r=1}^{\infty} R_{T}(r) \cos \left[\frac{\pi r g m}{m_{e} \cos (\Theta)}\right] \frac{(-R)^{r}}{r^{2}} J_{0}\left(\frac{4 \pi r t}{\hbar \omega_{c}}\right) \\
& \times \cos \left(\frac{2 \pi r \mu}{\hbar \omega_{c}}\right)
\end{aligned}
$$

is its quantum part with the conventional temperature, $R_{T}(r)=\left(2 \pi^{2} r k_{B} T / \hbar \omega_{c} \sinh \left(2 \pi^{2} r k_{B} T / \hbar \omega_{c}\right)\right.$, and Dingle collision damping factor $R$, as derived in Ref. $13\left(0<R_{T}(r)\right.$, $R \leqslant 1)$. Differentiating $\Omega$ with respect to the magnetic field at constant $\mu$, one obtains the oscillating part of the magnetization, $\widetilde{M}=-\partial \widetilde{\Omega} / \partial B$,

$$
\tilde{M}=\frac{e \mu \cos (\Theta)}{2 \pi^{2} \hbar d} \sum_{r=1}^{\infty} \frac{(-R)^{r} R_{T}(r)}{r} J_{0}\left(\frac{4 \pi r t}{\hbar \omega_{c}}\right) \sin \left(\frac{2 \pi r \mu}{\hbar \omega_{c}}\right),
$$

where we neglect small terms of the order of $2 t / \mu \ll 1$, and take $g=0$ for more transparency.

We are interested in the regime $\hbar \omega_{c} \ll 4 \pi t$, where threedimensional corrections to the spectrum are significant, rather than in the opposite ultraquantum limit, ${ }^{13}$ where the quantized spectrum is almost 2D. In our intermediate-field regime, one can replace the Bessel function in Eq. (5) by its asymptotic, $J_{0}(x) \approx(2 / \pi x)^{1 / 2} \cos (x-\pi / 4)$ at large $x$ to obtain

$$
\begin{aligned}
\tilde{M}= & \frac{e \mu \cos (\Theta)}{4 \pi^{2} \hbar d}\left(\frac{2 B}{\pi B_{\perp}}\right)^{1 / 2} \sum_{r=1}^{\infty} \frac{(-R)^{r} R_{T}(r)}{r^{3 / 2}}\left[\sin \left(\frac{2 \pi r F_{l}}{B}-\frac{\pi}{4}\right)\right. \\
& \left.+\sin \left(\frac{2 \pi r F_{s}}{B}+\frac{\pi}{4}\right)\right]
\end{aligned}
$$

where $B_{\perp} \equiv 4 \pi m t / e \hbar \cos (\Theta) \gg B$.

Naturally, the FT of Eq. (6) yields two fundamental frequencies in the grand-canonical ensemble, where $\mu$ is fixed (Fig. 2). However, the chemical potential oscillates with the magnetic field in the canonical system, ${ }^{1,6}$ which affects quantum corrections to magnetization. Using $n_{e}=-\partial \Omega / \partial \mu$, one can find the oscillating component, $\tilde{\mu} \equiv z \omega_{c} / 2 \pi=\partial \widetilde{\Omega} / \partial \mu$, of the chemical potential, $\mu=\mu_{0}+\tilde{\mu}$, where $\mu_{0}=d \pi \hbar^{2} n_{e} / m$ is its zero-field value and

$$
\begin{aligned}
z= & \left(\frac{B}{2 \pi B_{\perp}}\right)^{1 / 2} \sum_{r=1}^{\infty} \frac{(-R)^{r} R_{T}(r)}{r^{3 / 2}}\left[\sin \left(r z+\frac{2 \pi r f_{l}}{B}-\frac{\pi}{4}\right)\right. \\
& \left.+\sin \left(r z+\frac{2 \pi r f_{s}}{B}+\frac{\pi}{4}\right)\right] .
\end{aligned}
$$

Here, the "bare" fundamental frequencies, $f_{l, s}$ $=m\left(\mu_{0} \pm 2 t\right) / e \hbar \cos (\Theta)$, are now field independent. Remarkably, apart from a normalizing factor, the dimensionless quantum correction $z$ to the chemical potential [Eq. (7)] turns out identical to the magnetization quantum correction $\tilde{M}$ [Eq. (6)], which is not the case in a two-band canonical Fermi liquid. ${ }^{10}$
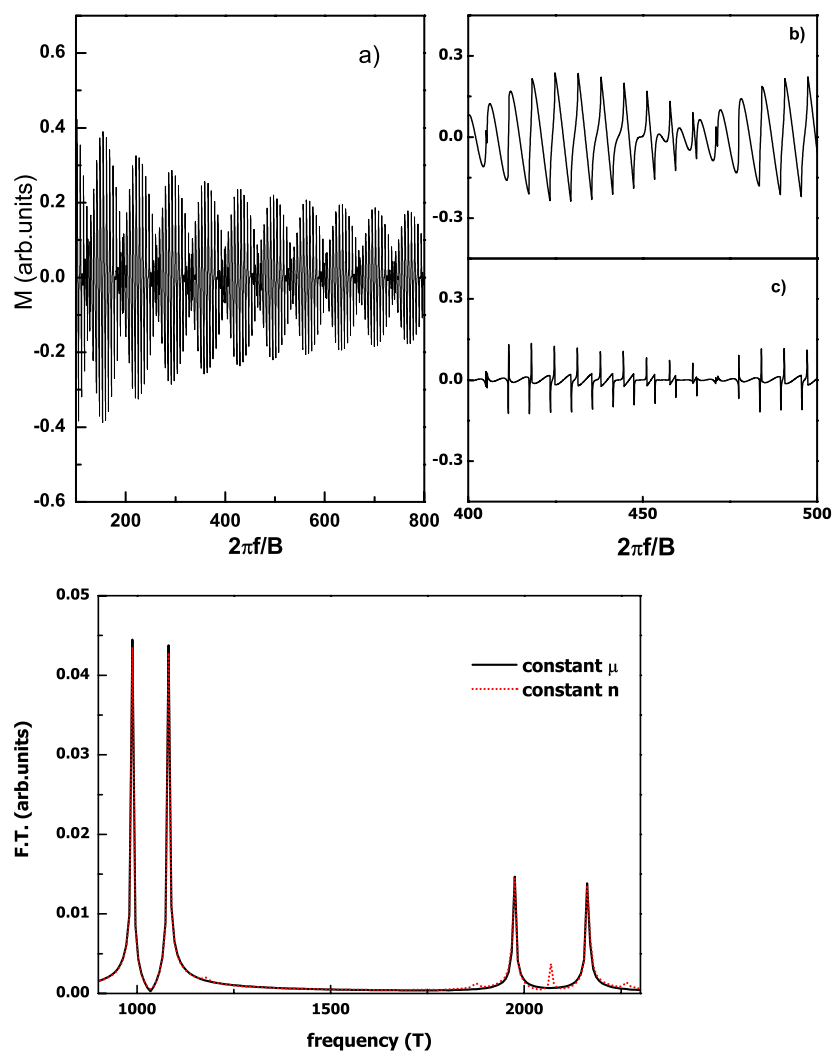

FIG. 2. (Color online) Upper panel: magnetization as functions of the inverse magnetic field $1 / B$ for (a) grand-canonical and (b) canonical single-band Fermi liquids, and (c) their difference. Lower panel: magnetization FTs for grand-canonical (solid line) and canonical (dashed line) single-band Fermi liquids. Here, $2 \pi f$ $=\pi\left(f_{l}+f_{s}\right)=6500 \mathrm{~T}, B_{\perp}=300 \mathrm{~T}, R=1$, and $T=g=0$.

To get insight regarding the FT of $z(B)$ or $\tilde{M}(B)$ [Eq. (7)], we first apply an analytical perturbation approach of Refs. 10 and 12 expanding $z$ in powers of $R$ up to the second order, $z \approx z_{1}+z_{2}+z_{\text {mix }}$, where

$$
\begin{aligned}
z_{1}= & -R R_{T}(1)\left(\frac{B}{2 \pi B_{\perp}}\right)^{1 / 2}\left[\sin \left(\frac{2 \pi f_{l}}{B}-\frac{\pi}{4}\right)\right. \\
& \left.+\sin \left(\frac{2 \pi f_{s}}{B}+\frac{\pi}{4}\right)\right]
\end{aligned}
$$

yields two first fundamental harmonics with the frequencies $f_{l}$ and $f_{s}$ identical to those of the grand-canonical system,

$$
\begin{aligned}
z_{2} \approx & \frac{R^{2} R_{T}(2)}{2^{3 / 2}}\left(\frac{B}{2 \pi B_{\perp}}\right)^{1 / 2}\left[\sin \left(\frac{4 \pi f_{l}}{B}-\frac{\pi}{4}\right)\right. \\
& \left.+\sin \left(\frac{4 \pi f_{s}}{B}+\frac{\pi}{4}\right)\right]
\end{aligned}
$$

yields two second fundamental harmonics with the frequencies $2 f_{l}$ and $2 f_{s}$ as in the grand-canonical system, and 


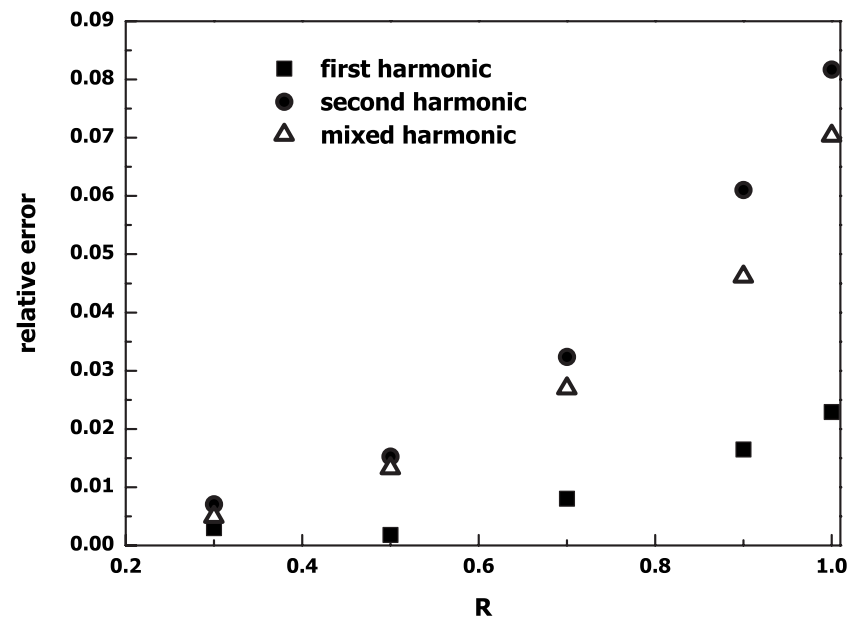

FIG. 3. Relative errors of analytical harmonic amplitudes [Eqs. (8)-(10)] with respect to numerically exact amplitudes.

$$
z_{m i x}=R^{2} R_{T}^{2}(1) \frac{B}{2 \pi B_{\perp}} \sin \left(\frac{2 \pi F_{+}}{B}\right)
$$

is the mixed harmonic with the frequency $F_{+}=f_{l}+f_{s}$, which is a specific signature of the canonical ensemble. Its amplitude is small compared with the first-harmonic amplitudes as $\left(B / 2 \pi B_{\perp}\right)^{1 / 2}$ in contrast with multiband systems, where the mixed-harmonic amplitudes have roughly the same order of magnitude as the fundamental-harmonic amplitudes at $R$ $=R_{T}(r)=1 .^{6,12}$ Also, there is no $F_{-}=f_{l}-f_{s}$ frequency in the FT spectrum of the single-band canonical system, different from the multiband canonical systems. ${ }^{7,8}$

To assess an accuracy of the analytical approximation [Eq. (10)] and some experimental conditions, allowing for resolution of the mixed harmonic, we present numerically exact magnetization and their FTs in Fig. 2 at $T=0$. Since convergence of the sum in Eq. (7) is poor at $T=0$, one can use its integral representation in numerical calculations as

$$
\begin{aligned}
z= & \left(\frac{B}{2 \pi B_{\perp}}\right)^{1 / 2} \Im\left[e^{-i \pi / 4} L i_{3 / 2}\left(-R e^{i\left(z+2 \pi f_{l} / B\right)}\right)\right. \\
& \left.+e^{i \pi / 4} L i_{3 / 2}\left(-R e^{i\left(z+2 \pi f_{s} / B\right)}\right)\right],
\end{aligned}
$$

where $L i_{3 / 2}(x)=\left(2 / \pi^{1 / 2}\right) \int_{0}^{\infty} d t t^{1 / 2} /\left(e^{t} / x-1\right)$ is the polylogarithm. The analytical amplitudes [Eqs. (8)-(10)] prove to be practically exact with the relative error below $10 \%$ at any $R$ (Fig. 3), as the amplitudes of the analytical theory of dHvA effect in canonical multiband systems. ${ }^{10,12}$ Another important feature of the numerical FT of the solution of Eq. (11) is that the resolution of the mixed central peak in the middle between two fundamental second harmonics [Fig. 2 (lower panel)] essentially depends on the magnetic-field window used in FT (Fig. 4). Since the mixed amplitude is relatively small as $\left(B / 2 \pi B_{\perp}\right)^{1 / 2} \ll 1$, the window affects its experimental resolution. We believe that a relatively small interval of the magnetic fields, used in FT, has prevented so far the single-band combination frequency to be seen in layered metals. $^{2-4,16}$ Importantly, since the characteristic field, $B_{\perp}$ $\propto J_{0}\left[k_{F} d \tan (\Theta)\right] / \cos (\Theta)$, is an oscillating function of the

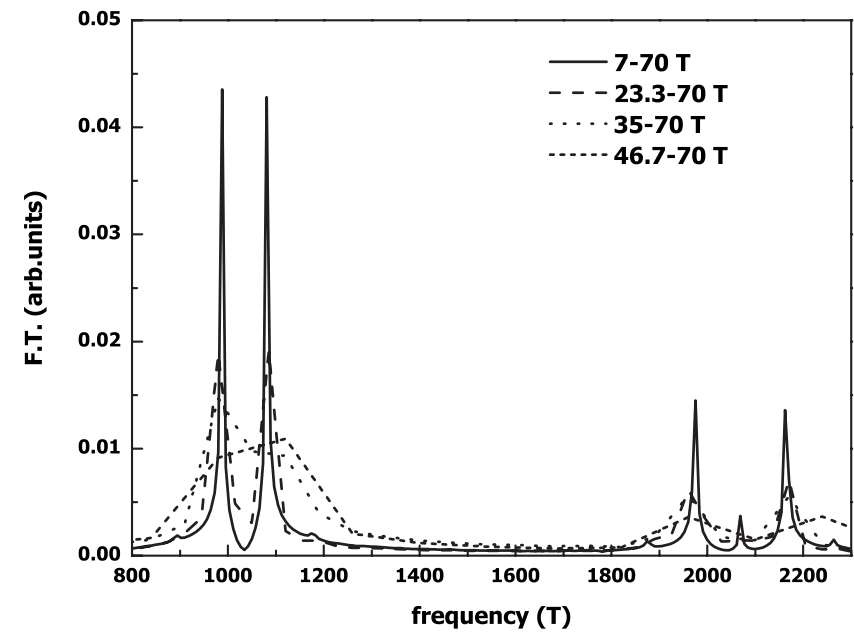

FIG. 4. Effect of the magnetic field window on the Fourier transform of magnetization. Decreasing the window increases the width of FT harmonics obscuring the mixed harmonic.

tilting angle $\Theta$, the combination amplitude also oscillates as a function of the angle, which could be instrumental in its experimental identification. We notice that the angle dependence of the second fundamental harmonics has been clearly observed in $\mathrm{Sr}_{2} \mathrm{RuO}_{4}{ }^{16}$

There is also mixing in the $\mathrm{SdH}$ quantum oscillations of transverse and longitudinal conductivities. For example, the longitudinal conductivity is given by the Kubo formula, ${ }^{20}$

$$
\left.\sigma=-\pi \hbar e^{2} \int d E \frac{\partial f(E)}{\partial E} \operatorname{Tr}\left[\delta(E-H) v_{\perp} \delta(E-H) v_{\perp}\right)\right]
$$

where $v_{\perp}=2 t d \sin \left(k_{\perp} d\right) / \hbar$ is the longitudinal component of the velocity operator, $H$ is the single-particle Hamiltonian including the impurity scattering, and $f(E)=1 /[\exp (E$ $\left.-\mu) / k_{B} T+1\right]$ is the Fermi-Dirac distribution function. Averaging over random impurity distributions and approximating the scattering rate by a constant $\Gamma$, one obtains the trace in Eq. (12) as $\left[v_{\perp} \Im\left[E-E_{n}\left(k_{\perp}\right)-i \Gamma\right]^{-1}\right]^{2}$ in the ladder approximation. Then, applying Poisson's summation, one can readily obtain a quantum correction $\widetilde{\sigma}$ to the classical conductivity (for detail see Refs. 21-23), which is (at $T=0$ )

$$
\widetilde{\sigma} \propto B \sum_{r=1}^{\infty} \frac{(-R)^{r}}{r} J_{1}\left(\frac{4 \pi r t}{\hbar \omega_{c}}\right) \cos \left(\frac{2 \pi r \mu}{\hbar \omega_{c}}\right) .
$$

The asymptotic of the Bessel function, $J_{1}(x)$ $\approx(2 / \pi x)^{1 / 2} \cos (x+\pi / 4)$, yields FTs of $\widetilde{\sigma}$ very similar to those of magnetization (Fig. 2), with the combination harmonic in the canonical system. Generally, the scattering rate depends on the magnetic field, ${ }^{22}$ so that its oscillations require more thorough analysis of the $\mathrm{SdH}$ effect, but mixing should be robust. Interestingly, some mixing of fundamental frequencies may occur even in grand-canonical multi- or single-band layered systems, if there is an interband or interextremal cross-section scattering by impurities. 
In conclusion, we have found the combination frequency in the quantum magnetic oscillations of the single-band canonical layered Fermi liquid. The difference between quantum oscillations of the canonical and grand-canonical ensembles is tiny (Fig. 2), but not obscured by the MB effect, which is absent in the single-band case in contrast with the multiband systems. We have also shown that the analytical (perturbation) FT amplitudes are numerically accurate even at zero temperature and in clean samples (i.e., for $R=1$ ) as they are in the multiband analytical theory. ${ }^{10,12} \mathrm{~A}$ wide magnetic-field window is essential for experimental resolution of the combination $\mathrm{dHvA} / \mathrm{SdH}$ frequency.

We greatly appreciate valuable discussions with Iorwerth Thomas and support of this work by EPSRC (UK) (Grant No. EP/D035589).
${ }^{1}$ D. Schoenberg, Magnetic Oscillations in Metals (Cambridge University Press, Cambridge, 1984).

${ }^{2}$ J. Singleton, Rep. Prog. Phys. 63, 1111 (2000).

${ }^{3}$ M. V. Kartsovnik, Chem. Rev. (Washington, D.C.) 104, 5737 (2004) and references therein.

${ }^{4}$ A. P. Mackenzie, S. R. Julian, A. J. Diver, G. J. McMullan, M. P. Ray, G. G. Lonzarich, Y. Maeno, S. Nishizaki, and T. Fujita, Phys. Rev. Lett. 76, 3786 (1996).

${ }^{5}$ J. M. Lifshits and A. M. Kosevich, Zh. Eksp. Teor. Fiz. 29, 730 (1955) [Sov. Phys. JETP 2, 636 (1956)].

${ }^{6}$ A. S. Alexandrov and A. M. Bratkovsky, Phys. Rev. Lett. 76, 1308 (1996).

${ }^{7}$ M. Nakano, J. Phys. Soc. Jpn. 66, 19 (1997).

${ }^{8}$ A. S. Alexandrov and A. M. Bratkovsky, Phys. Lett. A 234, 53 (1997).

${ }^{9}$ T. Champel, Phys. Rev. B 65, 153403 (2002); 69, 167402 (2004).

${ }^{10}$ A. S. Alexandrov and A. M. Bratkovsky, Phys. Rev. B 69, 167401 (2004).

${ }^{11}$ K. Kishigi and Y. Hasegawa, Phys. Rev. B 65, 205405 (2002); 72, 045410 (2005).

${ }^{12}$ A. S. Alexandrov and A. M. Bratkovsky, Phys. Rev. B 63, 033105 (2001).

${ }^{13}$ A. M. Bratkovsky and A. S. Alexandrov, Phys. Rev. B 65, 035418 (2002).

${ }^{14}$ J. Y. Fortin, E. Perez, and A. Audouard, Phys. Rev. B 71, 155101
(2005).

${ }^{15}$ R. A. Shepherd, M. Elliott, W. G. Herrenden-Harker, M. Zervos, P. R. Morris, M. Beck, and M. Ilegems, Phys. Rev. B 60, R11277 (1999).

${ }^{16}$ E. Ohmichi, Y. Maeno, and T. Ishiguro, J. Phys. Soc. Jpn. 68, 24 (1999).

${ }^{17}$ N. Harrison, J. Caulfield, J. Singleton, P. H. P. Reinders, F. Herlach, W. Hayes, M. Kurmoo, and P. J. Day, J. Phys.: Condens. Matter 8, 5415 (1996); P. S. Sandhu, Ju H. Kim, and J. S. Brooks, Phys. Rev. B 56, 11566 (1997); J. H. Kim, S. Y. Han, and J. S. Brooks, ibid. 60, 3213 (1999); S. Y. Han, J. S. Brooks, and J. H. Kim, Phys. Rev. Lett. 85, 1500 (2000); V. M. Gvozdikov and M. Taut, Phys. Rev. B 75, 155436 (2007); D. Vignolles, A. Audouard, V. N. Laukhin, J. Beard, E. Canadell, N. G. Spitsina, and E. B. Yagubskii, Eur. Phys. J. B 55, 383 (2007).

${ }^{18}$ Y. Kurihara, J. Phys. Soc. Jpn. 61, 975 (1989).

${ }^{19}$ K. Yamaji, J. Phys. Soc. Jpn. 58, 1520 (1989).

${ }^{20}$ R. Kubo, H. Hasegava, and N. Hashitsume, J. Phys. Soc. Jpn. 14, 56 (1959).

${ }^{21}$ T. Champel and V. P. Mineev, Phys. Rev. B 66, 195111 (2002); 67, 089901 (2003).

${ }^{22}$ P. D. Grigoriev, Phys. Rev. B 67, 144401 (2003).

${ }^{23}$ A. S. Alexandrov and V. V. Kabanov, Phys. Rev. Lett. 95, 076601 (2005); 95, 169902 (2005). 